Original Article

\title{
Curative Effects of Different Sequences of Vessel Interruption During the Completely Thoracoscopic Lobectomy on Early Stage Non-Small Cell Lung Cancer
}

\author{
Fengwei Li, MD, ${ }^{1,2}$ Guanchao Jiang, MD, ${ }^{1}$ Yingtai Chen, MD,${ }^{2}$ and Jun Wang, $\mathrm{MD}^{1}$
}

\begin{abstract}
Objective: To study the correlation between prognosis and different sequences of pulmonary artery and vein interruption during completely thoracoscopic lobectomy for early stage non-small cell lung cancer.

Methods: Retrospective analysis of 334 cases underwent completely thoracoscopic lobectomy, which were identified as stage I II non-small cell lung cancer by pathology. They were divided into three groups according to the order of vessel interruption: pulmonary vein first (Group V, $\mathbf{n}=174$ ), pulmonary artery first (Group $A, \mathbf{n}=93$ ), and artery-vein-artery group (Group $M, n=67$ ). Their preoperative and operative conditions, and the postoperative survival, recurrence were compared.

Results: Group A had less cases with history of smoking but more with history of pulmonary infection. The average bleeding amount during the operation in Group A is significantly less Group V, and Group $M$ fell in between them. The duration of operation and postoperative complications were similar among the three groups. The types of tumor recurrence were also similar, which were mostly distant metastasis. There was no statistically significant difference in tumor-free survival and overall survival among the three groups.

Conclusions: For the treatment of stage I III non-small cell lung cancer using completely thoracoscopic lobectomy, pulmonary artery interruption first can reduce the bleeding amount without affecting the operative difficulty and postoperative complications. The sequence of vessel interruption during lobectomy by thoracoscopic surgery would not affect tumor recurrence, metastasis and survival.
\end{abstract}

Keywords: non-small cell lung cancer, thoracoscopic lobectomy, vessel interruption, sequence

${ }^{1}$ Department of Thoracic Surgery, People's hospital of Peking University, Beijing, China

${ }^{2}$ Department of Thoracic Surgery, Beijing Aerospace General Hospital, Beijing, China

Received: February 9, 2015; Accepted: July 22, 2015

Corresponding author: Jun Wang, MD. Department of Thoracic Surgery, People's Hospital of Peking University, No. 11, Xizhimen South Street, Xicheng District, Beijing 100044, China Email: 254103852@qq.com

(C)2015 The Editorial Committee of Annals of Thoracic and Cardiovascular Surgery. All rights reserved.
Surgery is still the best treatment for early lung cancer currently, but approximately $40 \%-50 \%$ of patients will develop distant metastases after the operation. It was reported through pulmonary vein during an operation that remote dissemination may occur. ${ }^{1)}$ How to improve surgical methods and reduce the incidence of distant metastases and lower mortality is the direction of the continuous efforts of thoracic surgeons. Of note, whether different sequences of pulmonary artery and vein interruption during the operation have different effects on the metastatic dissemination of carcinomas remains controversial. 
Most surgeons interrupt the vein first believing that it will reduce tumor cells dissemination due to crushing of the tumor during the operation, which is more in line with the principle of tumor-free surgery. ${ }^{2)}$ However, others claimed that artery interruption first may reduce the extravasated blood in the remaining lung and the amount of bleeding. ${ }^{3)}$ There were also a few reports showing that the two methods had the similar effect on cancer dissemination. $\left.{ }^{4}\right)$

These studies were limited in that all pulmonary lobectomies were conducted by thoracotomy, with relatively short follow-up time and fewer cases. Other limitations included lack of stratified analysis, too many interference factors in the control group and non-specific tumor markers used. As a result, the conclusions remain to be further confirmed.

The completely thoracoscopic lobectomy began in the early 1990s. With over ten years of development and practice, its advantages including safety, feasibility and efficacy for treatment of early lung cancer has been appreciated. This technology has gradually become one of the main methods of lung cancer surgery.,5) This study retrospectively analyzed the effects of different sequences of pulmonary artery and vein interruption on stage I $\sim$ II non-small cell lung cancer in the completely thoracoscopic lobectomy conducted between September 2006 and June 2013 in our hospital. We concluded that the sequence of vessel interruption did not affect the prognosis of lung cancer, and the sequence of vessel interruption should be performed based on the specific circumstances of the individual patient.

\section{Materials and Methods}

\section{Cases}

Retrospective analysis was performed with 1337 patients underwent lobectomy in the thoracic surgery department between September 2006 and June 2013. Patients inclusion criteria were: (1) received the completely thoracoscopic lobectomy; (2) pathologically confirmed I II non-small cell lung cancer; (3) performed by the surgeons with more than 30 completely thoracoscopic lobectomy experience. Exclusion criteria were: (1) converted to open lobectomy; (2) lung metastatic cancer; (3) small cell lung cancer; (4) Pre-pulmonary wedge resection; (5) closed chest; (6) death during the operation. Based these criteria, we excluded open lobectomy (135 cases), lung metastatic cancer (39 cases), small cell lung cancer (20 cases), benign diseases ( 234 cases). Among the 909 non-small cell lung cancer left, we further excluded the stage 0 , III, IV (185 cases), pre-pulmonary wedge resection (284 cases), sleeve lobectomy (6 cases), composite lobectomy (28 cases), transiting open thoracotomy (41 cases), closed chest ( 8 cases), the operation performed by the surgeon with less than 30 cases experience (22 cases), death during the operation ( 1 case). Finally, a total of 334 cases met the selection criteria. According to the order of the pulmonary artery and vein interruption, they were divided into pulmonary vein first (Group V) with 174 cases, artery first group (Group A) with 93 cases, and mixed group (Group M) with 67 cases.

\section{Operation}

Group V: after a incision is made, the pulmonary vein was separated and interrupted first, followed by other vascular operation. Group A: the pulmonary artery was separated and interrupted first, and then the pulmonary vein. Group M: (1) In the process of right upper lobectomy, the sequence goes as follows: the posterior segment of arteryvein-the anterior apical segments of artery; (2) In the process of left upper lobectomy, the sequence goes as follows: the lingual and posterior artery-vein-the anterior apical segments of artery.

These three methods were all the complete thoracoscope lobectomy with systematic mediastinal lymph node dissection, including at least the seventh group and three groups from mediastinal lymph nodes and three from intrapulmonary lymph nodes. All lymph nodes and adjacent adipose tissue were removed together. Samples were marked according to the IASLC (2009) (The International Association for the Study of Lung Cancer) method after pathological examination by more than two senior pathologist respectively. The patients in the post-operative stage II or stage IB with high risk received the platinum-based double drugs chemotherapy for 1-4 periods, and then were followed up by every three months in the first year and every six months in the next years. Chest CT (Computed Tomography) and abdominal ultrasound were conducted in order to evaluate whether there are local recurrences, distant metastasis. The whole body bone scan was applied for the patients who were over 1 year after operation or had bone pain and other signs of bone metastases. Skull CT was also done for those who were over 1 year after operation or had discomfort in head to exclude intracranial metastasis.

\section{Statistical analysis}

The observed indexes related to operation included: operation time, intra-operative bleeding volumes, the difference of hemoglobin value between the first day after the 
Table 1 Patient clinical features $(\bar{x} \pm s /(\%))$

\begin{tabular}{|c|c|c|c|c|}
\hline & Group V $(\mathrm{n}=174)$ & Group A $(n=93)$ & Group M (n=67) & $P$-value \\
\hline Gender (male, $\%$ ) & $94(54.0)$ & $36(38.7)$ & $37(55.2)$ & 0.040 \\
\hline Age (year) & $62.8 \pm 10.8$ & $62.6 \pm 10.5$ & $60.8 \pm 9.8$ & 0.369 \\
\hline History of smoking $(\mathrm{n}, \%)$ & $69(39.7)$ & $23(24.7)$ & $28(41.8)$ & 0.026 \\
\hline Patient with pulmonary infections $(\mathrm{n}, \%)$ & $8(4.6)$ & $12(12.9)$ & $1(1.5)$ & 0.008 \\
\hline Pre-operation complications (n,\%) & $102(58.6)$ & $56(60.2)$ & $40(59.7)$ & 0.966 \\
\hline History of malignancy (n,\%) & $7(4.0)$ & $8(8.6)$ & $4(6.0)$ & 0.304 \\
\hline Lesion diameter $(\mathrm{cm})$ & $2.9 \pm 1.6$ & $2.8 \pm 1.4$ & $3.1 \pm 4.3$ & 0.732 \\
\hline Elevate pre-operative CEA (n, \%) & $27(15.5)$ & $8(8.6)$ & $10(14.9)$ & 0.267 \\
\hline Lesion location & & & & 0.000 \\
\hline Right upper lobe & $76(62.8)$ & $12(9.9)$ & $33(27.3)$ & \\
\hline Right middle lobe & $27(73.0)$ & $9(24.3)$ & $1(2.7)$ & \\
\hline Right lower lobe & $17(27.9)$ & $43(70.5)$ & $1(1.6)$ & \\
\hline Left upper lobe & $41(58.6)$ & $3(4.3)$ & $26(37.1)$ & \\
\hline Left lower lobe & $13(28.9)$ & $26(57.8)$ & $6(13.3)$ & \\
\hline \multicolumn{5}{|l|}{ Pre-operation pulmonary function (\% Pre) } \\
\hline FEV1 & $2.4 \pm 0.6$ & $2.2 \pm 0.6$ & $2.5 \pm 0.7$ & 0.277 \\
\hline FEV1/FVC & $76.5 \pm 8.4$ & $75.5 \pm 8.5$ & $78.5 \pm 11.1$ & 0.274 \\
\hline FEV1/pre & $82.7 \pm 14.9$ & $84.2 \pm 15.9$ & $88.3 \pm 15.5$ & 0.055 \\
\hline
\end{tabular}

CEA: carcinoembryonic antigen; FEV1: forced expiratory volume in one second; FVC: forced vital capacity

operation and pre-operation, postoperative drainage volumes, complications and the length of postoperative hospital stay, et al. The effectiveness of thoracoscopic pulmonary lobectomy was evaluated through comparing the tumorfree survival, the long-term survival, recurrence and metastasis between the two groups. Enumeration data were analyzed by the $\chi^{2}$ test. Quantitative data were analyzed by the Student's t test. Survival analysis adopted KaplanMeier method, Log-rank test and Cox regression model for single-factor and multiple factors analysis. Differences were considered significant when $P<0.05$.

\section{Results}

\section{General}

There were 334 cases in the three groups, with 167 male and 167 female, age between 33 to 86 (the middle age 63). Two-side upper lobectomy and right middle lobectomy were more prevalent in the Group V, while two-side lower lobectomy were more prevalent in the Group A. Group M mainly included a small number of two-side upper lobectomy. Group A was composed with more female, while other two groups (V and M) with more male. Group A had less cases with history of smoking but more with history of pulmonary infection. The patient's age, main complications, tumor history, lesion diameter, pre-operative tumor markers and pre-operative lung function (Forced expiratory volume in one second (FEV1), FEV1/Forced vital capacity (FEV1/FVC) and Fev1/predicted Fev1 (FEV1/ pre)) had no statistically significant differences among the three groups $(P>0.05$, Table 1$)$.

\section{The perioperative period}

All 334 cases underwent successful operation without serious complications, 80 cases with minor complications including prolonged drainage time ( $>7$ days), atrial fibrillation, pulmonary infection, subcutaneous emphysema and chylothorax, et al. Except for two cases of chylothorax which were cured by reoperation of ligation of thoracic duct and local exudates treatment and one case of chest wall hematoma which was removed by reoperation, others were cured by conservative treatment.

The mean operation time was $181.7 \pm 53.5 \mathrm{~min}$, and the mean bleeding amount was $128.0 \pm 123.0 \mathrm{~mL}$. There were no statistical differences between individual surgeon's levels among the three groups. The bleeding amount during the operation in Group A was less than that in Group V while that in Group $\mathrm{M}$ fell in between. The difference was statistically significant $(P<0.05)$. But there were no statistically significant difference in the operation time, the hemoglobin value between the first day after the operation and pre-operation, required 
Table 2 The perioperative period characters of the three groups $(\bar{x} \pm s /(\%))$

\begin{tabular}{|c|c|c|c|c|}
\hline & Group V $(\mathrm{n}=174)$ & Group A $(n=93)$ & Group M $(n=67)$ & $P$-Values \\
\hline Operations by senior surgeon (n, \%) & $125(71.8)$ & $60(64.5)$ & $38(56.7)$ & 0.073 \\
\hline Operation time (min) & $186.2 \pm 53.6$ & $167.0 \pm 45.2$ & $190.5 \pm 62.5$ & 0.797 \\
\hline Bleeding amount (mL) & $148.3 \pm 142.9$ & $105.1 \pm 97.5$ & $107.0 \pm 123.0$ & 0.005 \\
\hline Hemoglobin variation & $12.5 \pm 8.0$ & $10.2 \pm 7.1$ & $12.9 \pm 10.3$ & 0.802 \\
\hline Required blood transfusion (n, \%) & $7(4.0)$ & $3(3.2)$ & $1(1.5)$ & 0.663 \\
\hline Drainage time (day) & $6.6 \pm 3.0$ & $5.8 \pm 3.4$ & $6.3 \pm 3.3$ & 0.293 \\
\hline Hospital stay time (day) & $9.5 \pm 9.9$ & $8.0 \pm 4.0$ & $8.7 \pm 4.2$ & 0.270 \\
\hline Complications (n, \%) & $44(25.3)$ & $23(24.7)$ & $13(19.4)$ & 0.625 \\
\hline Pathological types (n, \%) & & & & 0.826 \\
\hline Squamous cell carcinoma & $32(18.4)$ & $15(16.1)$ & $9(13.4)$ & \\
\hline Adenocarcinoma & $134(77.0)$ & $75(80.6)$ & $54(80.6)$ & \\
\hline Others* & $8(4.6)$ & $3(3.2)$ & $4(6.0)$ & \\
\hline Differentiation degree (n, \%) & & & & 0.467 \\
\hline Highly differentiated & $23(13.2)$ & $16(17.2)$ & $11(16.7)$ & \\
\hline Moderately differentiated & $86(49.4)$ & $52(55.9)$ & $31(47.0)$ & \\
\hline Poorly differentiated & $65(37.4)$ & $25(26.9)$ & $24(36.4)$ & \\
\hline TNM stages (n, \%) & & & & 0.477 \\
\hline I & $138(79.3)$ & $79(84.9)$ & $56(83.6)$ & \\
\hline II & $36(20.7)$ & $14(15.1)$ & $11(16.4)$ & \\
\hline
\end{tabular}

TNM: tumor-node-metastasis classification

blood transfusion ratios, postoperative closed thoracic drainage tube indwelling time, postoperative hospital stay, minor complications, tumor pathological types, differentiation degree and stages $(P>0.05$, Table 2$)$.

\section{Long-term results}

Median follow up was 30 (4-86 months) months in Group V, with 4 loss in 174 cases. Six patients had local recurrence; one patient had cutting edge swelling considering as local recurrence because of enhanced metabolism on PET-CT scan (Positron Emission TomographyComputed Tomography); five cases had enlarged hilar and mediastinal lymph nodes; and 22 cases had distant metastases by imaging examination (11 pulmonary metastases, five bone metastases, three brain metastases, three liver metastases). Median follow up was 26 (11-80 months) months in Group A, with five loss in 93 cases. Two cases showed local recurrence suggested by enlarged hilar and mediastinal lymph node on CT scan; seven cases had distant metastases by imaging examination (four pulmonary metastases, two pleural metastases, one bone metastases). In Group M, median follow up was 20 months (7-77 months), with three loss in 67 cases. One patient had local recurrence indicated by enlarged hilar and mediastinal lymph nodes on CT scan; two cases had distant metastases by imaging examination (one pulmonary and one bone metastases).
No statistically significant differences were found in the local recurrence and distant metastases among the three groups $(P>0.05)$. There were also no statistically significant differences in the 1-, 3- and 5-year overall survival and tumor-free survival $(P>0.05$, Figs. 1, 2). No statistically significant differences were found as well by stratified analysis according to the excision sites, gender, history of smoking and pulmonary infection. Variables having a significant impact on overall survival by univariate Cox regression analysis were age, elevated preoperative CEA (carcinoembryonic antigen) and postoperative stages $(P<0.05)$. Further multivariate Cox regression analysis showed that age and postoperative stages still had statistically significant $(P<0.05)$ (Table 3).

\section{Discussion}

By retrospective analysis of patients underwent thoracoscopic lobectomy in our hospital, we discussed the curative effects of different sequences of pulmonary artery and vein interruption during the completely thoracoscopic lobectomy on early stage non-small cell lung cancer. The three groups had significant differences in the distribution of lesions. It is mainly because that compared to the traditional thoracotomy, completely thoracoscopic lobectomy can reveal and zoom in certain areas that are difficult to do 


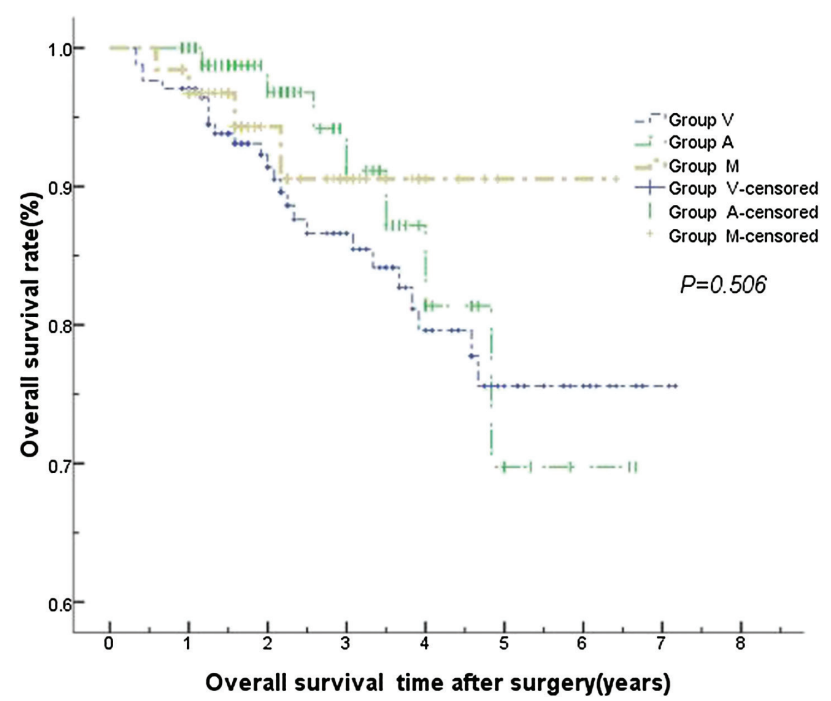

Fig. 1 There were no statistically significant differences in overall survival time among the three groups.

in open thoracotomy. However, this technique has limitations. Unlike open thoracotomy, it is impossible to separate and dissect from various angles in the completely thoracoscopic lobectomy when serious adhesion appears. It is also difficult to stop bleeding by directly pressing. Therefore, forced dissection from one direction or a certain fixed order may cause serious bleeding, which then requires the conversion to open thoracotomy. Sometimes, there are swellings and calcified lymph nodes around pulmonary veins, which make it difficult to separate the pulmonary veins first. Under this situation, if the lobar fissure is dissected and the pulmonary artery branches are handled first, it will be easier to deal with the pulmonary veins from another angle. This condition is especially prominent in the operation under endoscope. For patient safety, there is possibly only one vessel interruption order for a certain pulmonary lobe of a certain patient. We summarized the experience of over 500 thoracoscopic lobectomy cases and optimized this approach. One of the core principles is to do the easy ones first instead of following the traditional fixed interruption order of artery after vein. ${ }^{7)}$ As a result, the safety of the thoracoscopic lobectomy was improved. To be specific, the rate of conversion to open thoracotomy has decreased; the duration of the surgery has shortened and the bleeding amount has reduced. For this reason, except for the vein first group and artery first group, there were still some cases in mixed group in the study. And three groups vary in the distribution of lesion locations.

The three groups showed differences in the gender distribution, smoking history and the history of pulmonary

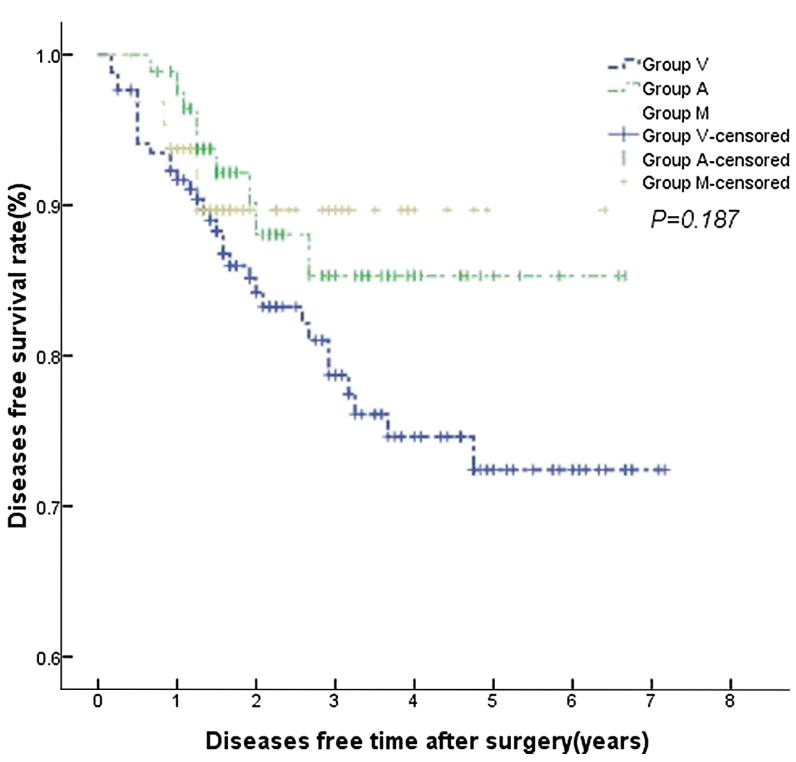

Fig. 2 There were no statistically significant differences in diseases free time among the three groups.

infection. But this study mainly discussed the effects of vessel interruption order on the survival and surgical operation. it has indicated that these differences has no influence on the survival and recurrence through stratified analysis and Cox model analysis. Smoking and pulmonary infection may theoretically aggravate the adhesion in thoracic cavity, which can increase the amount of bleeding and the difficulty of surgery. However, this study excluded the extreme situation, closed chest, of these effects. Therefore, no correlation has been found when the history of smoking and pulmonary infection was used as influence factors to analyze the bleeding amount and surgery duration.

Traditionally, the vein should be cut before the artery in the pulmonary lobectomy for lung cancer treatment, which was thought that may reduce the dissemination of tumor cells during the operation. ${ }^{2}{ }^{2}$ By comparing the CEA level before and after pulmonary lobectomy, Kurusu found that surgical operation could raise the detection rate of peripheral tumor cell. ${ }^{2)}$ The change was even more obvious in the artery cut first group. By univariate Cox model analysis, the study found that the increased opreoperative CEA in the peripheral blood was a risk factor, but further multifactor analysis indicated that this risk factor had no statistical significance. This study was a retrospective study, so detection of circulating tumor cell was not conducted. Similar to this study, Wulf Sienel detected the circulating tumor cell in the pulmonary vein of 49 lung cancer patients using cytokeratin-associated antigens as the marker. The patients 
Table 3 Cox regression analysis of survival

\begin{tabular}{lcccccccc}
\hline & & & & & & & \multicolumn{2}{c}{$95 \%$ CI } \\
\cline { 6 - 9 } & B & SE & Wald & df & Sig. & RR & Low & Up \\
\hline pTNM & -1.341 & 0.339 & 15.675 & 1 & 0.000 & 0.261 & 0.135 & 0.508 \\
CEA & 0.055 & 0.018 & 8.926 & 1 & 0.003 & 1.056 & 1.019 & 1.095 \\
\hline
\end{tabular}

CI: confidence interval; B: beta, equal to regression coefficient; SE: standard error; df: degree of freedom; RR: relative risk; pTNM: pathological TNM classification; CEA: carcinoembryonic antigen

were divided into two groups: tumor cell positive (10 cases) and tumor cell negative (39 cases). Follow-up study showed that free circulating cell positive was a risk factor of prognosis, but the effects of vessel process on survival was not compared in the study. ${ }^{8}$ Yael Refaely compared the vein cut first group (133 cases) and the artery cut first group (146 cases) but found no significant difference in recurrence and survival between the two groups. ${ }^{4)}$ Kozak conducted the prospective randomized study on the effects of artery and vein interruption sequences. The study compared the artery cut first group ( 215 cases) with the vein cut first group (170 cases) and found that there's no statistical difference in recurrence and survival between the two groups. ${ }^{9)}$ However, these studies all focused on thoracotomy lobectomy. Kurusu pointed out that the traction of pulmonary tissue increased the risk of recurrence and metastases. ${ }^{2)}$ Yamashita held the view that thoracoscopic surgery had a more powerful traction on pulmonary lobe and the tumor. The detection rate of peripheral tumor cell was higher, which might have effects on the survival after surgical treatment. ${ }^{10)}$ Our study, for the first time, reveals that different sequences of pulmonary artery and vein interruption during completely thoracoscopic lobectomy has no influence on the recurrence, metastases, tumor-free survival and overall survival. Tumor metastases involve a complex interaction between tumor cells and host cells. The tumor cells shedding from the tumor discontinuously enter the blood circulation where most of them are recognized and eliminated by multiple immune factors and immune cells. A few tumor cells can break through the vascular endothelium but the majority of them will be destructed by immune system in the tissues and organs. Eventually very few cells can settle in distal tissues and develop into metastatic colonization.

There are also some scholars who support the idea that the artery should be cut first, and then the vein. ${ }^{2,3)}$ They think that this order can reduce the extravasated blood in the remaining lung and the amount of bleeding.
Pulmonary congestion can lead to the enlargement of the pulmonary volume and make the narrow intrathoracic room even smaller, which, therefore, extends the duration of surgery and increases the operation difficulty. This is more important for thoracoscopic operation. In this study, the average amount of bleeding in Group A was $105.1 \mathrm{ml}$, which was obviously lesser than that in Group V (148.3 ml). Group M fell in between the above two groups with the number of $107.0 \mathrm{ml}$, which showed that cutting the artery first can reduce the bleeding volume during the operation. However, the advantage of this less bleeding did not present in the change of hemoglobin before and after the surgery or the ratio of transfusion. It also has no influence on complications or postoperative recovery indicators like the time with closed thoracic drainage tube. Both $105.1 \mathrm{ml}$ and $148.3 \mathrm{ml}$ are less than $5 \%$ of the total blood volume, so it will not affect the health condition of the body. Surgical duration is an important manifestation of the difficulty of the surgery. The average duration of operation in Group A was about 167.0 min, which was less than the $186.2 \mathrm{~min}$ in Group V. However, the difference showed no statistical significance. On the other hand, it indicates that, instead of situations that require complicated operation such as difficulty in vessel treatment and arteriorrhexis, the increase of bleeding caused by cutting the vein first mainly comes from oozing blood of pulmonary wound.

There are several factors that may affect the postoperative distant metastasis of tumor. When selecting the cases, we tried our best to exclude a variety of main affecting factors. The study excluded the patients with stage III and above as there was a higher possibility of micrometastasis before the operation, which may have a significant influence on the postoperative distant metastasis. The study excluded patients who received the wedge resection of tumor and the follow-up lobectomy with a confirmed pathology. The pulmonary wedge resection has an obvious compress on the pulmonary tissue and the tumor, which 
may result in the shedding of tumor cells into the blood. Moreover, there is no tumor in the follow-up operation and the vessel cut order will not influence the long-term recurrence, metastases and survival. So these kind of patients were excluded to reduce the confounding factors. Their tumors are usually small and peripheral, so we excluded most patients with small tumors. Together with the exclusion of patients with the stage III and above, the diameter of the tumor was limited to a small range (on average $3 \mathrm{~cm}$ ). Smaller diameter dispersion can explain that the tumor diameter is not an independent risk factor in the survival analysis.

The limitations of this study are as follows. First of all, as a retrospective study, certain deviation appears during the selecting of the cases. The amount of cases was small with a large time span. The patients were mainly in the early or middle stage, so the follow-up was short and most of them did not experience the outcome event. Therefore, the conclusion needs further follow-up validation. This article aimed to VATS lobectomy and pathological early stage non-small cell lung cancer patients. Indeed there were staging improving in Clinical practice, but of small probability, ${ }^{11)}$ such patients have more disturbing factors of tumor spread and metastasis. Such patients may have some slightly isolated metastases which were difficult to detect preoperative. Once a postoperative metastasis come up, it is difficult to tell whether it was caused by different sequences of vascular interruption or proliferation of original micrometastases. Similarly with the relevant literature, patient with staging improving remains comparatively rare in our center. And patients' clinical data were not balanced between the two groups, our existing data can not be used to make conclusions about this condition. Therefore in order to ensure our persuasion pathological early stage non-small cell lung cancer patients were restricted. Most of the cases in vein cut first group were middle or upper lobectomy while most of the cases in artery cut first group were lower lobectomy. There were no analysis of postoperative effects caused by surgical complexity, such as adhesion of regional lymph nodes or long separation time. These factors need further investigation by conducting prospective study.

\section{Conclusions}

In conclusion, for thoracoscopic treatment of I $\sim$ II stage non-small cell lung cancer, handling and cutting artery first can reduce the amount of bleeding during the surgery without affecting the operative difficulty and postoperative complications. The sequence of vessel interruption during lobectomy by thoracoscopic surgery would not affect tumor recurrence, metastasis and survival. The sequence of vessel interruption should be performed based on the specific circumstances of the individual patient.

\section{Consent}

Ethics approval of People's hospital of Peking University ethics committee was obtained.Written informed consent was obtained from the patient for the publication of this report and any accompanying images.

\section{Authors' Contributions}

FWL carried out data analysis and wrote the manuscript; GCJ and JW made the operations; YTC conducted the statistical analyses; GCJ drafted the manuscript; All authors read and approved the final approval of the manuscript.

\section{Acknowledgements}

We would like to thank Xizhao Sui, MD, Xiao Li, MD and Feng Yang, MD for reviewing imaging and medical record. We also would like to thank Kejun Han, $\mathrm{PhD}$ for helping in translation, Yun Wang for collecting patient data.

\section{Disclosure Statement}

All authors received no funding in any part from any source. The authors declare that they have no competing interests.

\section{References}

1) Cho Y, Hida Y, Kaga K, et al. Brain metastases secondary to tumor emboli from primary lung cancer during lobectomy. Ann Thorac Surg 2008; 86: 312-3.

2) Kurusu Y, Yamashita J, Hayashi N, et al. The sequence of vessel ligation affects tumor release into the circulation. J Thorac Cardiovasc Surg 1998; 116: 107-13.

3) Yellin A, Sadetzki S, Simansky DA, et al. The sequence of vessel interruption during lobectomy: does it affect the amount of blood retained in the lobe? Eur J Cardiothorac Surg 2007; 31: 711-3.

4) Refaely Y, Sadetzki S, Chetrit A, et al. The sequence of vessel interruption during lobectomy for non-small cell lung cancer: is it indeed important? J Thorac Cardiovasc Surg 2003; 125: 1313-20.

5) Yan TD, Black D, Bannon PG, et al. Systematic review and meta-analysis of randomized and nonrandomized trials on safety and efficacy of video-assisted thoracic surgery lobectomy for early-stage non-small-cell lung cancer. J Clin Oncol 2009; 27: 2553-62. 
6) Wang J, Jiang G. Video-assisted thoracic surgery lobectomy on early stage non-small cell lung cancer: another opportunity of thoracic surgeon. Chinese J of Clinical Thorac and Cardiovasc Surg 2008; 24: 145-6.

7) Li Y, Wang J, Sui XZ, et al. Operative technique optimization in completely thoracoscopic lobectomy: Peking University experience. Chinese J of Clinical Thorac and Cardiovasc Surg 2010; 26: 300-6.

8) Sienel W, Seen-Hibler R, Mutschler W, et al. Tumour cells in the tumour draining vein of patients with nonsmall cell lung cancer: detection rate and clinical significance. Eur J Cardiothorac Surg 2003; 23: 451-6.

9) Kozak A, Alchimowicz J, Safranow K, et al. The impact of the sequence of pulmonary vessel ligation during anatomic resection for lung cancer on long-term survival - a prospective randomized trial. Adv Med Sci 2013; 58: 156-63.

10) Yamashita JI, Kurusu Y, Fujino N, et al. Detection of circulating tumor cells in patients with non-small cell lung cancer undergoing lobectomy by video-assisted thoracic surgery: a potential hazard for intraoperative hematogenous tumor cell dissemination. J Thorac Cardiovasc Surg 2000; 119: 899-905.

11) Watanabe A, Mishina T, Ohori S, et al. Is video-assisted thoracoscopic surgery a feasible approach for clinical $\mathrm{N} 0$ and postoperatively pathological N2 non-small cell lung cancer? Eur J Cardiothorac Surg 2008; 33: 812-8. 